\title{
A QUALITY ASSURANCE PROTOCOL FOR RADIOCARBON DATING LABORATORIES
}

Compiled by AUSTIN LONG, with advice and consent from many colleagues Department of Geosciences, The University of Arizona, Tucson, Arizona 85721 (Additional comments are welcome)

The purpose of this Quality Assurance (QA) protocol is to summarize guidelines that have been accepted by directors of many radiocarbon dating laboratories throughout the world, and by the International Atomic Energy Agency (IAEA). Some laboratories have followed similar procedures successfully for years. Laboratories that carefully adhere to this protocol will produce consistently reliable data that will be comparable in accuracy to all other laboratories following this or any other equally rigorous quality assurance program. This statement does not, however, pertain to samples with ${ }^{14} \mathrm{C}$ activities highly sensitive to method or degree of pretreatment, as pretreatment techniques vary among laboratories.

Laboratories following this protocol may state in data reports that "These analyses were performed according to the quality assurance protocol approved by the Glasgow Intercomparison Workshop, September, 1989." Radiocarbon date end users should understand that a specific procedure cannot guarantee the accuracy of a date, as a variety of factors, many of which are beyond the laboratory's control, can affect accuracy. Also, many laboratories and directors have developed unique procedures that yield highly accurate ${ }^{14} \mathrm{C}$ results. The intent here is not to disturb successful individuality, rather to correct false complacency. Some laboratories may choose to adopt only some of these suggested guidelines. Some laboratories, depending on personnel, volume of data output, and precision required may modify the number of Quality Assurance samples processed and the time intervals between replications.

BASIC ELEMENTS OF THE QUALITY ASSURANCE (QA) PROTOCOL

I. Written Procedures

II. Sample Documentation, Traceability

III. Analysis of Primary Standards

IV. Replication of Quality Assurance Samples

V. Regular Intercomparisons Using Natural (Unknown Activity) Samples

VI. Recognition and Correction of Problems

VII. Establishment of Total Analytical Precision

I. Written Procedures

A. An up-to-date procedures notebook, containing detailed steps with diagrams of equipment, must be in the laboratory while the analysis is underway.

B. Records must indicate the nature and dates of all changes in procedures, replacement, repair, modification and adjustment of equipment.

II. Sample Documentation, Traceability

A. It should be possible for anyone who is unfamiliar with the laboratory, using the laboratory's written and computer records, to reconstruct what happened to any sample, 
when it happened, and who did it, from the sample's arrival to the report of the data and the ultimate disposition of the remains of the sample.

1. Log Book. All samples, upon arrival, must be logged-in with a sequential number. The log book will contain the sample lab number, an identification code the submitter gave it, a brief description of the physical or chemical nature of the sample, the name of the submitter, and the date of arrival in the lab. The lab number will follow the sample through the lab. Some laboratories have found it useful for a sample information sheet to accompany the sample throughout the lab. This not only adds redundancy to help prevent confusion of samples, but is a handy reference aid at different stages of the procedure, and serves as a note pad for technical comments.

2. Procedures. The lab personnel will keep up-to-date records of all operations performed on each sample (for example, type of pretreatment performed, comments on pretreatment, $\mathrm{CO}_{2}$ yields, benzene yields, counter performance, purity corrections, age calculation details, and copies of relevant correspondence). These records should be in permanently-kept notebooks, and also on the sample information sheets (above) kept on file.

3. Sample Archival. Remaining sample material, if any, should either be kept in laboratory, returned to submitter or discarded after an established length of time. Each laboratory must have a policy on sample archival, and maintain records of the final disposition of each sample.

B. A minimum of primary records should be kept in perpetuity. This minimum is the information required for publication in RADIOCARBON or the International Radiocarbon Data Base (IRDB), plus laboratory processing data and counting and calculation summary. The lab notebooks should be archived as well as the sample information sheets. The latter are useful for quick reference, as they contain an encapsulation of all that happened to the samples in the ${ }^{14} \mathrm{C}$ dating lab. Some laboratories even retain primary count-rate data.

C. Laboratories should retain primary counting data for samples and the graphs of standards and backgrounds (or blanks, in the case of AMS) as long as the particular analysis equipment is in service, and for at least five years after the data appear in publication.

\section{Analysis of Counting Background, Chemical Blanks and Primary Standards}

A. Establish the count rates of background and NBS Oxalic Acid at regular intervals and immediately after replacement, repair, modification and adjustment of measurement equipment. For AMS, the chemical blank is more critical than machine background, and measurement of known activity standard with each loading (wheel) is normal procedure for adjusting for variations in transmission efficiency.

B. Time intervals between routine measurements of background and NBS primary standard will vary with general stability of equipment, and frequency of measurement of quality assurance samples (see below).

C. Plots of these data $( \pm 10)$ should be on calendric scales, with annotations explaining adjustments of equipment or procedures, that accompanied aberrations and discontinuities in the linearity of the plot. Annotations will also explain adjustments in data (for example, atmospheric pressure corrections, purity compensation). These graphs will be available to illustrate the system's reliability. 


\section{Replication of Reference Materials}

A. Four QA sample materials will soon be available from the IAEA along with the results of several laboratories' analyses of these materials. It is recommended that laboratories analyze these materials on a regular basis, at least annually, as a regular check on system accuracy (Scott, Long \& Kra 1990).

B. The amount of these reference materials will be limited and each lab should produce its own in-house reference samples in bulk, which can be cross-calibrated to the IAEA materials, and to the NBS oxalic acid standards. The type of in-house reference materials should be appropriate to each lab's major interests, and should be analyzed more frequently than the IAEA samples. After the first dating of the IAEA samples in 1990, the consensus ages will be widely reported (thus they will become of known age).

C. The purposes of repeat analysis of these known-age materials at regular intervals are:

1. Continual monitoring of analytical accuracy without pretreatment (except for Two Creeks wood)

2. Recognition of analytical problems before they propagate to the release of erroneous data

3. Establishment of analytical precision of procedures

D. Technicians will regularly run each of these samples through each combustion/hydrolysis/ purification/catalysis/counting system in the laboratory. Frequency and types and ages of these QA monitors will depend on the precision sought and ages of samples the laboratory normally runs. One of the younger QA materials should be run more often in the case of new equipment or personnel in order to establish analytical precision and maintain accuracy. New or modified equipment should be tested more frequently until steady operation is proven.

E. Under routine operation, about $20 \%$ of counting time should be devoted to quality assurance activity (background, primary standard, secondary standards). Change in equipment, procedures or personnel will temporarily require a more intensive quality assurance effort. Some well-established high-precision $\beta$-counting and AMS labs devote up to $30 \%$ counting time on precision and accuracy test samples.

\section{Regular Intercomparisons Using Natural (Unknown Activity) Samples}

As part of the proposals for Quality Assurance and designed to provide an objective and independent check on analytical accuracy and precision, regular intercomparisons will continue to be organized on an international basis by the SURRC-based team in the UK. A crucial feature of the comparisons will be the use of natural sample materials, requiring pretreatment, results of which will not be known in advance.

Laboratories should participate in these programs which will be run every 2 or 3 years, and a key component of which will be the "help" offered to participating laboratories. These labs will be encouraged to publicize their performance in the program.

Participation in these international programs is not intended to replace any recognized need for and/or benefit from ad hoc intercomparisons organized on an individual basis. 


\section{Recognition and Correction of Problems}

A. All QA analyses should be plotted on calendric graphs and examined for deviations beyond statistical expectation of the established ${ }^{14} \mathrm{C}$ age. A practical alternative is the statistical control graph approach of Switsur (this issue).

The results of the blind intercomparisons (Section V) as reported to the organizers should be tabulated and made available.

B. Frequent analysis of background and laboratory QA materials should reveal problems before affected dates are released. The IAEA QA materials should be run occasionally to ensure proper calibration with "real" samples at all age ranges. Two-thirds of the data points should lie within one standard deviation of the accepted values, about evenly split above and below expected values. Significant departure from this expected pattern is an alert of problems.

C. Trouble-shooting is beyond the scope of these guidelines. However, considerable expertise is available within the ${ }^{14} \mathrm{C}$ dating community, and several of our most experienced lab directors have expressed surprise at never having been consulted for advice. This expertise could become widely available on an informal basis within the framework of the intercomparison program.

\section{Establishment of Total Analytical Precision}

A. Radiocarbon dating convention by $\beta$-counting (Stuiver $\&$ Polach 1977: 357) requires dates to be reported with the \pm figure reflecting not only the Poisson (approximated by Gaussian) 1o counting statistics of the sample, oxalic acid and background, but also "additional errors caused by inaccuracies in voltage, pressure, temperature, dilution, et cetera." The authors further assert that the \pm figure should include the uncertainty in the $\delta^{13} \mathrm{C}$, which does not significantly contribute to the uncertainty if actually measured. This \pm figure (combined counting statistics, or CCS) does not include variability introduced during all other steps in sample processing. In practice, only some laboratories adhere to this convention. Some laboratories arbitrarily increase this figure; AMS laboratories usually report an uncertainty based on replication of data, similar to the TAP defined below.

B. The error figure most relevant to the end user of ${ }^{14} \mathrm{C}$ dates is the Total Analytical Precision (TAP) obtained by repeat analysis, through the entire chemical and physical system in the laboratory, of a homogeneous material similar to many samples of unknown age normally run through the lab (ie, the samples assayed under the procedures of section IV). The TAP will be equal to or greater than the CCS. This variability of repeat analyses may not be Gaussian, but establishing whether it is might require a large number $(>100)$ of analyses. We recommend that $\beta$-counting laboratories report their TAP as well as their conventional CCS figure along with dates and pMC's. The TAP will be equal to or greater than the CCS, and may be different for different laboratory preparation or counting systems, and change with time.

C. Error Multiplier factor: It is impractical to evaluate the TAP for each range of \pm values, as even within the same laboratory systems, the \pm figure depends on the counting time and dilution factor, among other variables. In order to adjust the CCS to the TAP, some labs find it convenient to use an error multiplier factor (see Scott et al 1983; Stuiver \& Pearson 1986). As for the TAP, laboratories should re-evaluate and update this figure regularly. 
D. For $\beta$-counting systems: The Total Analytical Precision (TAP) is based on replicate ${ }^{14} \mathrm{C}$ analyses of the IAEA and in-house replicate reference materials. Results included in this calculation should be all those analyzed within the past year with the following exception. Do not include analyses originally affected by some analytical problem now recognized and corrected before release of erroneous data. The TAP is a continuously updated figure based on at least the last 20 replicates with close to the same "conventional combined counting statistics figure". Examine the grouping of these data graphically or statistically for symmetry and outliers. Look for trends with time. Compare the standard deviation of the group $\left(\sigma_{n-1}\right)$ with the average \pm figure (the combined counting statistics, or CCS, of Stuiver and Polach, 1977) of the individual dates. In properly functioning laboratories, the statistical groups will be symmetrical, data will show no trends with time, and the TAP will approach the "ideal" average CCS of the individual dates.

E. For AMS systems: A common procedure among AMS labs is to evaluate errors based on counting statistics and based on repeated analysis of the same target within a wheel loading. The \pm figure reported would be the larger of the two in terms of 1 standard deviation. Some AMS labs report an error on the age based on the statistical summation of all known sources of uncertainty, in a manner analogous to that Stuiver and Polach (1977) proposed for $\beta$-counting procedures.

We recommend that AMS laboratories report \pm values of their ${ }^{14} \mathrm{C}$ measurements based on reproducibility of test samples within a single loading of a batch of targets, and on repeat samples run over several months. A sensitive parameter to monitor is the ratio of ${ }^{14} \mathrm{C} /{ }^{13} \mathrm{C}$ 's of 2 reference materials with different ${ }^{14} \mathrm{C}$ contents (Linick et al 1986). This would be comparable to the Total Laboratory Precision in $\beta$-counting laboratories. Laboratories should specify how the error is calculated (see Donahue et al 1990).

F. Due to the availability of a variety of calibration schemes, we recommend that all modifications to the conventionally reported ${ }^{14} \mathrm{C}$ data be explicitly stated. All \pm values reported on uncalibrated ${ }^{14} \mathrm{C}$ measurements should be based on $1 \sigma$ standard deviation. Report $\delta^{13} \mathrm{C}$ along with ${ }^{14} \mathrm{C}$ date, and indicate whether $\delta^{13} \mathrm{C}$ is measured or estimated. If calibrated date is reported as well, give probability limit $(1 \sigma, 2 \sigma)$ and reference to calibration scheme.

\section{REFERENCES}

Donahue, DJ, Linick, TW and Jull, AJT 1990 Isotope-ratio and background corrections for accelerator mass spectrometry radiocarbon Measurements. Radiocarbon 32(2): 135-142.

Linick, TW, Jull, AJT, Toolin, LJ and Donahue, DJ 1986 Operation of the NSF-Arizona accelerator facility for radioisotope analysis and results from selected collaborative research projects. In Stuiver, $\mathrm{M}$ and $\mathrm{Kra}, \mathrm{RS}$, eds, Internatl ${ }^{14} \mathrm{C}$ conf, 12th, Proc. Radiocarbon 28(2A): 522-533.

Scott, EM, Long, A and Kra, RS, eds, 1990 International workshop of intercomparison of radiocarbon laboratories, Proc. Radiocarbon, this issue.

Scott, EM, Baxter, MS and Aitchison, TC 1983 An assessment of variability in radiocarbon dating methods of low-level counting and spectrometry (IAEA, Berlin, 1981): 371-392.

Stuiver, M and Pearson, GW 1986 High-precision calibration of the radiocarbon time scale, AD $1950-500$ BC. In Stuiver, $\mathrm{M}$ and Kra, RS, eds, Internatl ${ }^{14} \mathrm{C}$ conf, 12th, Proc. Radiocarbon 28(2B): 805-838.

Stuiver, M and Polach, HA 1977 Discussion: Reporting of ${ }^{14} \mathrm{C}$ data. Radiocarbon 19(3): 355-363.

Switsur, Roy 1990 Statistical quality control graphs in radiocarbon dating. Radiocarbon, this issue. 